\title{
The Molecular Ridge Close to 30 Doradus in the Large Magellanic Cloud
}

\author{
Jürgen Ott ${ }^{\mathrm{A}, \mathrm{B}, \mathrm{L}}$, Tony Wong ${ }^{\mathrm{C}}$, Jorge L. Pineda ${ }^{\mathrm{D}}$, Annie Hughes ${ }^{\mathrm{E}, \mathrm{F}}$, \\ Erik Muller $^{\mathrm{F}}$, Zhi-Yun Li ${ }^{\mathrm{G}}$, Min Wang ${ }^{\mathrm{H}}$, Lister Staveley-Smith ${ }^{\mathrm{I}}$, \\ Yasuo Fukui ${ }^{\mathrm{J}}$, Axel Wei ${ }^{\mathrm{K}}{ }^{\text {, Christian Henkel }}{ }^{\mathrm{K}}$, and Ulrich Klein ${ }^{\mathrm{D}}$ \\ A National Radio Astronomy Observatory, 520 Edgemont Road, Charlottesville, \\ VA 22903, USA \\ B California Institute of Technology, 1200 E. California Blvd., Caltech Astronomy \\ 104-25, Pasadena, CA 91125-2400, USA \\ ${ }^{C}$ Department of Astronomy, University of Illinois, 1002 W. Green St., Urbana, \\ IL 61801, USA \\ D Argelander Institut für Astronomie, Universität Bonn, Auf dem Hügel 71, \\ 53121 Bonn, Germany \\ ${ }^{\mathrm{E}}$ Centre for Supercomputing and Astrophysics, Swinburne University of Technology, \\ Hawthorn, VIC 3122, Australia \\ F CSIRO Australia Telescope National Facility, Cnr Vimiera \& Pembroke Roads, \\ Marsfield, NSW 2122, Australia \\ G Department of Astronomy, University of Virginia, PO Box 400325, Charlottesville, \\ VA 22903-4325, USA \\ H Purple Mountain Observatories, CAS, 2 West Beijing Road, Nanjing 210008, China \\ I School of Physics M013, University of Western Australia, Crawley, WA 6009, Australia \\ J Department of Astrophysics, Nagoya University, Furocho, Chikusaku, \\ Nagoya 464-8602, Japan \\ K Max-Planck-Institut für Radioastronomie, Auf dem Hügel 69, 53121 Bonn, Germany \\ ${ }^{\mathrm{L}}$ Corresponding author. Email: jott@nrao.edu
}

Received 2007 December 20, accepted 2008 May 10

\begin{abstract}
With the ATNF Mopra telescope we are performing a survey in the ${ }^{12} \mathrm{CO}(1-0)$ line to map the molecular gas in the Large Magellanic Cloud. For some regions we also obtained interferometric maps of the high density gas tracers $\mathrm{HCO}^{+}$and $\mathrm{HCN}$ with the Australia Telescope Compact Array. Here we discuss the properties of the elongated molecular complex that stretches about $2 \mathrm{kpc}$ southward from 30 Doradus. Our data suggest that the complex, which we refer to as the 'molecular ridge', is not a coherent feature but consists of many smaller clumps that share the same formation history. Likely triggers of molecular-cloud formation are shocks and shearing forces that are present in the surrounding south-eastern Hi overdensity region, a region influenced by strong ram pressure and tidal forces. The molecular ridge is at the western edge of the the overdensity region where a bifurcated velocity structure transitions into a single disk velocity component. We find that the ${ }^{12} \mathrm{CO}(1-0)$ and $\mathrm{HI}$ emission peaks in the molecular ridge are typically near each other but never coincide. A likely explanation is the conversion of warmer, low-opacity Hi to colder, high-opacity HI from which $\mathrm{H}_{2}$ subsequently forms. On smaller scales we find that very dense molecular gas, as traced by interferometric $\mathrm{HCO}^{+}$and $\mathrm{HCN}$ maps, is associated with star formation along shocked filaments and with rims of expanding shell-like structures, both created by feedback from massive stars.
\end{abstract}

Keywords: ISM: evolution — ISM: molecules — galaxies: ISM — galaxies: individual (Large Magellanic Cloud) — radio lines: ISM — galaxies: Magellanic Clouds

\section{Introduction}

The study of star formation (SF) in molecular clouds is an area which has seen rapid progress in recent years and the Magellanic Clouds are increasingly seen as one of the most important laboratories for this research. At a distance of about $50-60 \mathrm{kpc}$ and an inclination angle of $\sim 35^{\circ}$ (van der Marel \& Cioni 2001) for the Large Magellanic Cloud (LMC), current and future instruments can resolve individual star-forming regions on sub-parsec scales and, at the same time, assess the global picture on galactic scales. The Magellanic Clouds also contain a large range of very different environments that can 
be observed to test theories of star formation. The 30 Doradus (30 Dor) region in the LMC, for example, is the most vigorous site of current SF in the Local Group. Consequently, the surroundings of 30 Dor are dominated by a strong far-UV radiation field. Possible triggering of SF on expanding shells can be studied, for example, in the LMC4 region toward the northeast of the LMC (e.g. Braun et al. 1997; Yamaguchi et al. 2001; Cohen, Stavely-Smith \& Green 2003). In addition, the LMC and the Small Magellanic Cloud (SMC) are gravitationally interacting with each other and with the Galaxy (cf. the numerical models of, e.g., Murai \& Fujimoto 1980; Gardiner, Sawa \& Fujimoto 1994; Staveley-Smith et al. 2003; Connors, Kawata \& Gibson 2006), and the motion of the LMC through the Galactic halo causes strong ram pressure effects (e.g. de Boer et al. 1998a). It should be noted, however, that tidal or ram-pressure models alone cannot explain the full morphology and dynamics of the gas and stars in the Magellanic System, and a synthesis of both is likely required (Mastropietro et al. 2005). All of these external interactions, as well as factors internal to the LMC (e.g. shocks, density waves, and bar-induced motions) influence the ability to form stars on local and global scales and can potentially be studied in the Magellanic Clouds in great detail.

Also, with metallicities of $\sim 30 \%$ and $\sim 10 \%$ solar in the LMC and SMC, respectively, cooling and chemistry of gas in the Magellanic Clouds are clearly different than in the Galaxy and the conditions may resemble those at higher redshifts, at times when galaxies were generally less evolved than today. Another important property of the Magellanic Clouds and in particular the LMC is that the numbers of resolved giant molecular clouds with associated star formation is large. For example, Fukui et al. (1999) counted 107 molecular complexes in the 2.6 $(=37 \mathrm{pc})$ resolution NANTEN ${ }^{12} \mathrm{CO}(1-0)$ survey of the LMC. It is therefore possible to derive statistically meaningful results for a large sample of regions, all at the same distance and therefore resolution.

The location of 30 Dor and the surrounding massive atomic and molecular gas reservoir dominating the southeastern part of the LMC had led to many speculations on its structure and star-forming history. For example Fujimoto \& Noguchi (1990) and, more recently, Bekki \& Chiba (2007) were able to model this region based on dynamical and hydro-dynamical simulations of the LMCSMC interaction, not including the Galaxy. Gardiner, Turfus \& Putman (1998), on the other hand, attempted to simulate the amount of star formation based on the dynamics of the off-center bar in the LMC. Their simulations, though not designed to predict the gas distribution, do show that asymmetries can be induced by the bar. de Boer et al. (1998a) pointed out that the ages of structures in the LMC increase clockwise from the eastern 30 Dor region toward the north. They argued that the LMC may undergo SF triggered by a bow-shock at the eastern edge, where the ram pressure due to the motion of the LMC through the Galactic halo is expected to be greatest. The clockwise rotation of the LMC then turns the newly formed, but now aging, stellar populations toward the north. In other words, ram pressure creates a 'hot spot' of SF (currently 30 Dor) that remains in the eastern part of the LMC while the galaxy rotation brings fresh gas toward and newly formed stars away from it. If this interpretation is correct, one may infer that conditions upstream from 30 Dor, i.e. from the East toward the South, may be those encountered just before stars eventually form. This would be a very fortunate situation as it is usually difficult to predict the timescales and locations of future SF in galaxies.

It is common knowledge that stars form out of molecular gas, and indeed the region south of 30 Dor contains a remarkably straight, elongated $\mathrm{CO}$ structure which we refer to as the 'molecular ridge'. ${ }^{12} \mathrm{CO}(1-0)$ maps from the Columbia survey (Cohen et al. 1988), the NANTEN telescope (Fukui et al. 1999), and the SEST key project (Kutner et al. 1997; Johansson et al. 1998) show that, with a mass of $10^{7} \mathrm{M}_{\odot}$, about one third of all the molecular gas that is traced by $\mathrm{CO}$ is found in this structure stretching $\sim 2 \mathrm{kpc}$ south of 30 Dor. Johansson et al. (1998) provide an analysis of the N159 and 30 Dor regions and they find kinetic temperatures in the range $10-50 \mathrm{~K}$. Kutner et al. (1997) were able to describe the southern part of the molecular ridge but they restricted their analysis to a description of the general morphology of the feature and a comparison of line strength to those in Galactic clouds.

In this paper we present first results of a comprehensive, high angular resolution ${ }^{12} \mathrm{CO}(1-0)$ survey of the entire molecular ridge obtained with the ATNF Mopra ${ }^{1}$ telescope. The observations are the pilot for the MAGMA project ('The Magellanic Mopra Assessment') which is currently underway and which will provide high resolution ${ }^{12} \mathrm{CO}(1-0)$ maps of molecular clouds across the entire LMC and SMC, mapping all regions where NANTEN has detected emission, but with a beam size $\sim 16$ times smaller in area. In terms of sensitivity and resolution, MAGMA is comparable to the SEST key program but MAGMA encompasses all CO traced molecular clouds rather than a selected ensemble. In Section 2 we describe the observations and data reduction, which is followed by the presentation of our results in Section 3. A discussion of the results is provided in Section 4 and a summary in Section 5 .

\section{Observations and Data Reduction}

During the 2005 southern winter season, we observed the molecular ridge close to 30 Dor with the single dish Mopra telescope in the ${ }^{12} \mathrm{CO}(1-0)$ molecular transition (rest frequency $115.271 \mathrm{GHz}$ ). Mopra was used in the on-the-fly mapping mode and we observed about $\sim 1205 \times 5^{\prime}$ maps in two orthogonal scanning directions with 1.8 -h integration time each. The total time on source was therefore of

\footnotetext{
${ }^{1}$ The Mopra radio telescope is part of the Australia Telescope which is funded by the Commonwealth of Australia for operation as a National Facility managed by CSIRO.
} 


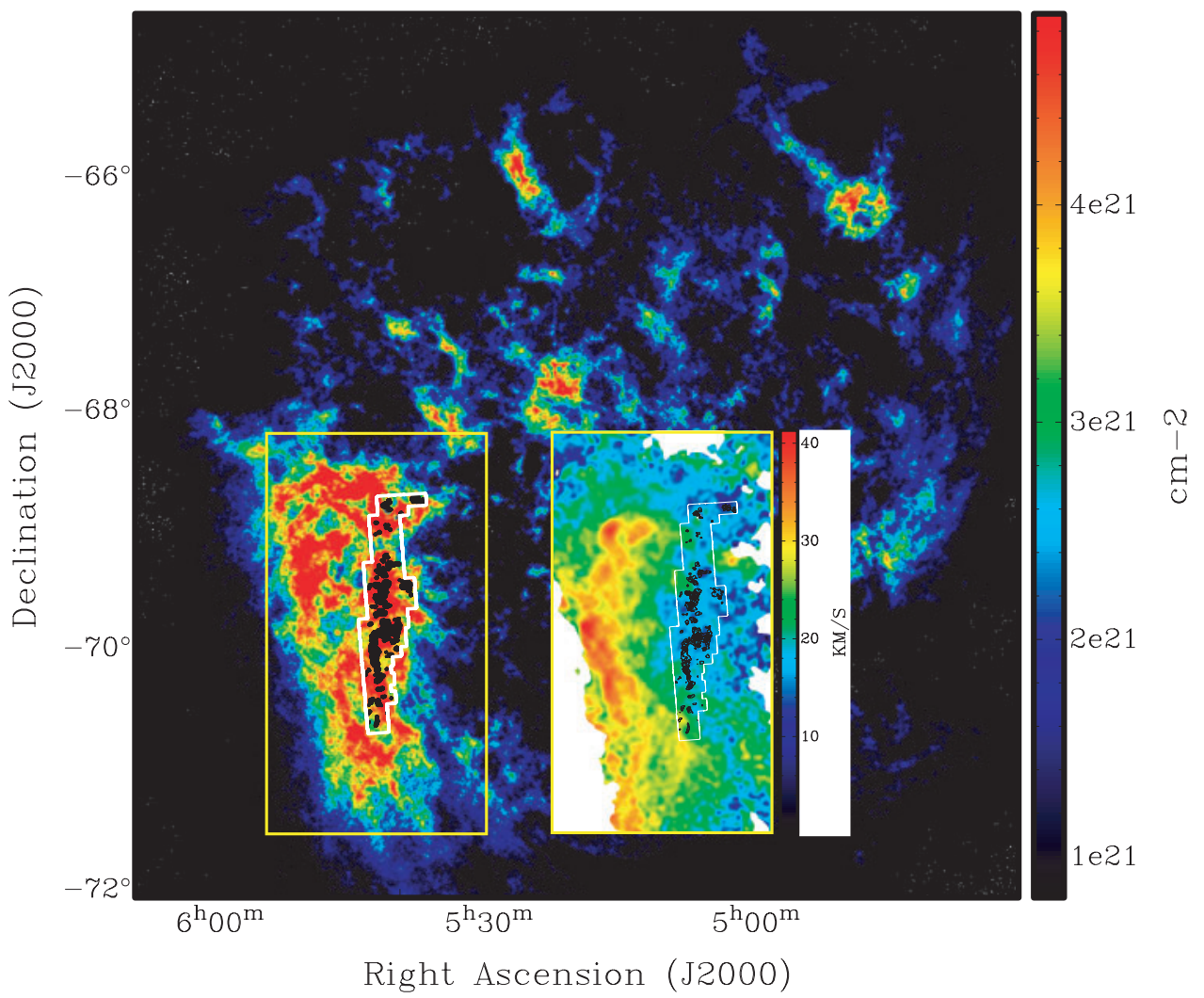

Figure 1 Hi column-density map of the LMC (taken from Kim et al. 1998, beam size $1^{\prime}$ ) with the contours of our molecular ridge CO data overlaid in black. The white polygon outlines the region observed. The yellow boxed insert is the same region as the one marked on the column density map but it displays the second moment of the HI data (for better signal-to-noise, this map was smoothed to three times the resolution of the integrated Hi map). Note that toward the south-east the second moment is dominated by the velocity difference of the disk and $L$ components rather than by the line widths of the neutral gas.

order $250 \mathrm{~h}$. The Mopra AT correlator was set up to observe at a bandwidth of $64 \mathrm{MHz}$ split into 1024 channels. This corresponds to a velocity coverage of $\sim 166 \mathrm{~km} \mathrm{~s}^{-1}$ and a resolution of $\sim 0.16 \mathrm{~km} \mathrm{~s}^{-1}$ and the spectral window was centered on a velocity of $230 \mathrm{~km} \mathrm{~s}^{-1}$ LSR. The observation of each map was preceded by pointing calibration on the bright $\mathrm{SiO}$ maser $\mathrm{R}$ Dor, with typical corrections less than $5^{\prime \prime} . T_{\mathrm{A}}^{*}$ system temperatures of the observations were usually $\sim 600 \mathrm{~K}$ and the data were calibrated against a warm absorber that was inserted every $\sim 30$ minutes. The final data set has a size of $\sim 0.6 \times 2.0^{\circ}$ which, at an assumed distance of the LMC of $50 \mathrm{kpc}$, corresponds to $0.5 \times 1.8 \mathrm{kpc}$, the long side along declination. A map of the survey region is shown in Figure 1. The natural spatial resolution of Mopra at $\sim 115 \mathrm{GHz}$ is about $35^{\prime \prime}$. The data were reduced with the ATNF package LIVEDATA to derive the quotient against line-free reference positions up to $\sim 0.5^{\circ}$ away from the observed fields. First-order baselines fitted to line-free channels were subtracted from the resulting spectra. All data were regridded to produce a position-position-velocity 3-dimensional data cube using GRIDZILLA. The 'inner' Mopra error beam is in the range of $40-80^{\prime \prime}$ which is similar to the size of the emission and for the analysis here we applied the extended beam efficiency of $\eta_{\mathrm{xb}}=0.55$ (Ladd et al. 2005) to convert $T_{\mathrm{A}}^{*}$ to extended beam brightnesses $T_{\mathrm{xb}}$. For better signal-to-noise ratios, the data were eventually smoothed to a resolution of $45^{\prime \prime}$, which corresponds to a physical length of $\sim 11 \mathrm{pc}$. The rms noise of the data is about $0.4 \mathrm{~K}$ in a $0.16-\mathrm{km} \mathrm{s}^{-1}$ velocity channel. A comparison with earlier SEST data at a similar resolution (Johansson et al. 1998) toward the molecular gas in N159 shows that the Mopra and SEST fluxes agree within $10 \%$.

\section{Results}

Maps of the molecular ridge derived from the ${ }^{12} \mathrm{CO}(1-0)$ data cube are displayed in Figure 2. The integrated intensity map clearly shows that the molecular ridge is a slim (width $\sim 100-200 \mathrm{pc})$ but long $(\sim 1.8 \mathrm{kpc})$ feature along the declination axis. The brightest clumps are toward the N159 region (see Figure 2) and about half a degree south of it at the 'kink' of the ridge. Between N159 and 30 Dor relatively little molecular gas is observed. Using the Strong et al. (1988) 'standard' Galactic COintensity to $\mathrm{H}_{2}$-column-density $\left(X_{\mathrm{CO}}\right)$ conversion factor of $2.3 \times 10^{20} \mathrm{~cm}^{-2}\left(\mathrm{~K} \mathrm{~km} \mathrm{~s}^{-1}\right)^{-1}$ the detection limit (3 consecutive channels with $3-\sigma$ detection each) is at about $1.3 \times 10^{20} \mathrm{~cm}^{-2}$ (corresponding to a mass of $\sim 200 \mathrm{M}_{\odot}$ within a beam) and for the molecular ridge we derive a total molecular gas mass of about $3.2 \times 10^{6} \mathrm{M}_{\odot}$. But note that studies of CO with the Columbia 1.2-m, the SEST 

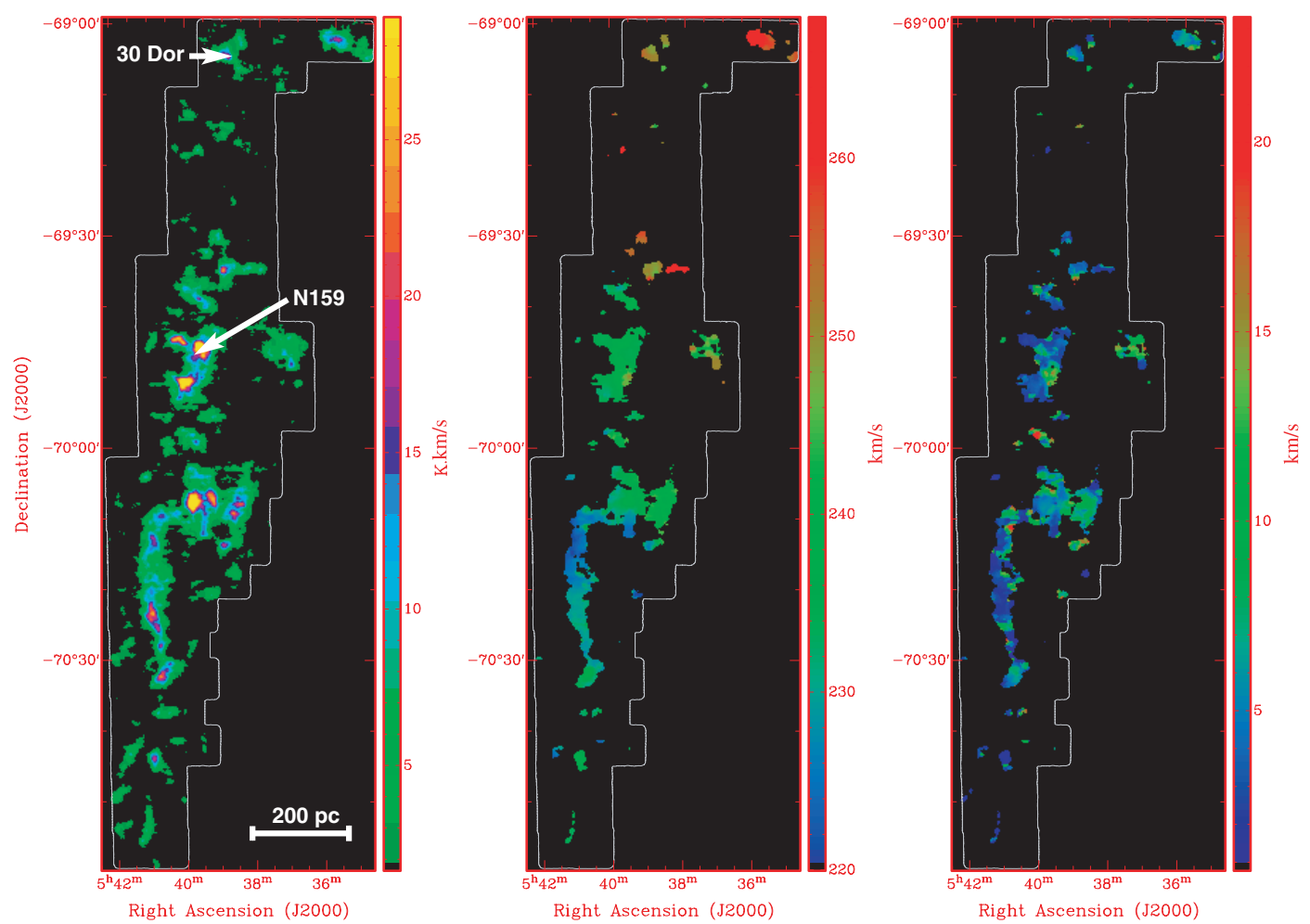

Figure 2 Mopra ${ }^{12} \mathrm{CO}(1-0)$ maps of the molecular ridge close to 30 Dor. From left to right: Integrated intensity in $\mathrm{K} \mathrm{km} \mathrm{s}^{-1}$, intensity-weighted velocities (moment 1), dispersions (moment 2).

and NANTEN telescopes have shown that the LMC $X_{\mathrm{CO}}$ factor may be a few times larger than this Galactic value (Cohen et al. 1988; Israel 1997; Fukui et al. 1999, but see Pineda et al. 2008). For their NANTEN survey data, Fukui et al. (1999) use $X_{\mathrm{CO}}$ of $9 \times 10^{20} \mathrm{~cm}^{-2}\left(\mathrm{~K} \mathrm{~km} \mathrm{~s}^{-1}\right)^{-1}$ and they derive a value of $4 \times 10^{7} \mathrm{M}_{\odot}$ for the entire molecular gas in the LMC. If there are no variations of $X_{\mathrm{CO}}$ within the LMC, the ratio of the fluxes should equal the ratio of masses and if we compare our values to those of NANTEN, we confirm that the molecular ridge contains about one third of the total molecular mass of the LMC.

\section{Discussion}

The very thin and long shape of the molecular ridge would argue for it to be a coherent structure. A comparison of the $\mathrm{HI}$ and $\mathrm{CO}$ velocities in various position-velocity diagrams along the ridge (Figure 3) shows, however, that the molecular clumps are not confined to a specific velocity component of the atomic gas. In addition, the molecular clumps are somewhat separated from each other, only connected by some faint common molecular envelope. This is even more obvious when the 3-dimensional position-position-velocity data cube is rotated around the declination axis. In a more global context the molecular gas in the ridge finds itself on the western edge of the south-eastern, high-column-density region in the LMC, at a position where the Hi column densities are still high. However, the HI emission is bifurcated in two velocity components, called the disk and ' $L$ ' component (Luks \& Rohlfs 1992). The velocity separation of the two components is very large towards the eastern edge of the LMC, but they start to merge into a single velocity component at about the position of the molecular ridge (see the second moment image in Figure 1). This suggests that the clumps in the molecular ridge were created by the same large-scale triggering event and thus have similar formation histories, not only in environmental terms but also on similar timescales.

\subsection{Molecular Cloud Formation}

In Figure 4, CO contours are overlaid over an integrated Hi column-density map. The CO peaks are usually found very close to Hi peaks but they virtually never coincide. Such a morphology was already indicated by the lower resolution NANTEN data $\left(\sim 2.6^{\prime}\right)$ and with the high angular resolution of Mopra, this is unambiguously confirmed. If, instead of the integrated Hi column density, the Hi peak flux is compared to the ${ }^{12} \mathrm{CO}(1-0)$ intensity, the peaks of the two gas tracers are getting slightly closer to each other but they still never coincide. To quantify this effect, pixelto-pixel correlations between the $\mathrm{CO}$ luminosity and the Hi column density and Hi peak brightness temperature are shown in Figure 5. The CO bright pixels are at intermediate Hi column densities and brightnesses and not at the largest values. In particular, the Hi peak brightness values where the brightest $\mathrm{CO}$ emission is observed hovers around 60 to $100 \mathrm{~K}$. In the following, we will discuss 

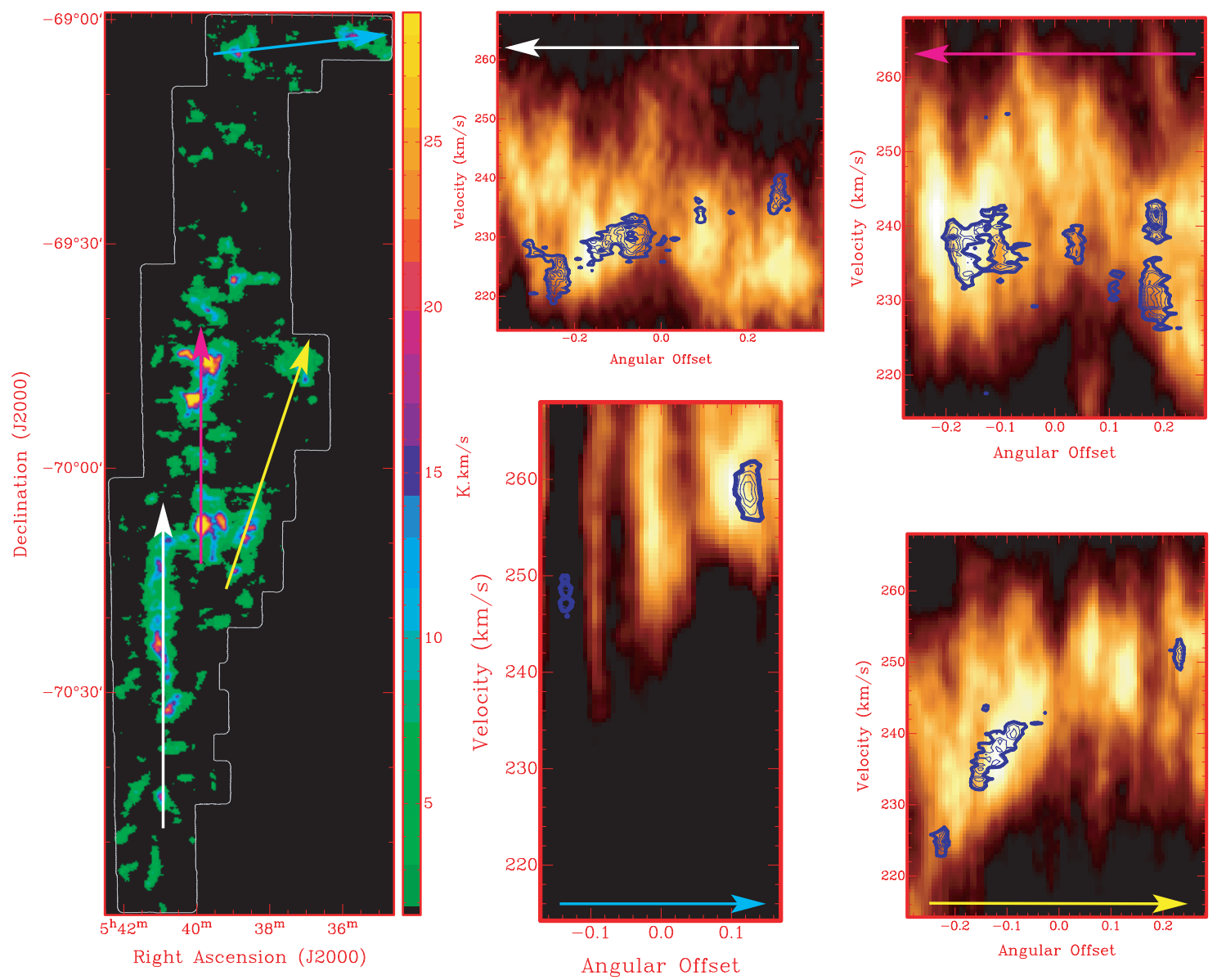

Figure 3 Position-velocity cuts through the $\mathrm{CO}$ and $\mathrm{HI}$ data cubes. The arrows in the left panel indicate the position, direction and length where the cuts are taken. The corresponding position-velocity plots are shown in the middle and to the right. Arrows of the same color belong together; they are also coded by letters 'A' to ' $\mathrm{D}$ '. The color scale is data from the HI cube and the blue contours are the CO data. Note that the $\mathrm{CO}$ at the 30 Dor region exhibits HI in absorption (start of cut 'C'). ATCA Hi observations are taken from Kim et al. (1998).

different mechanisms that may be responsible for the shift of the $\mathrm{CO}$ and $\mathrm{HI}$ emission peaks:

\subsubsection{Rapid Conversion of Warm $\mathrm{H}_{\mathrm{I}}$ into $\mathrm{H}_{2}$}

At a certain threshold, the atomic gas combines into molecular hydrogen and more than just the gas above the threshold is converted, creating slight depressions in the map of atomic HI. If the Hi gas remains optically thin one can determine the entire amount and distribution of hydrogen atoms from both, $\mathrm{HI}$ and $\mathrm{H}_{2}$ in the gas. In this scenario one would expect a smooth distribution of hydrogen, some in atomic, and some in molecular form. As an example we consider N159. The atomic gas in this region has typical column densities of $\sim 7 \times 10^{21} \mathrm{~cm}^{-2}$ whereas the HI at the positions of the CO peaks have a somewhat lower column of $N(\mathrm{HI}) \sim 5 \times 10^{21} \mathrm{~cm}^{-2}$. The CO peaks (smoothed to the resolution of the Hi map, 60") have intensities of $\sim 35 \mathrm{~K} \mathrm{~km} \mathrm{~s}^{-1}$. Converted with a Galactic $X_{\mathrm{CO}}$ factor, this becomes an $\mathrm{H}_{2}$ column density of $N\left(\mathrm{H}_{2}\right) \sim 8 \times 10^{21} \mathrm{~cm}^{-2}$ which adds to the aforementioned Hi column density of $\sim 5 \times 10^{21} \mathrm{~cm}^{-2}$. In total, the hydrogen column density at the positions of the molecular clouds is determined to be $N\left(\mathrm{H}_{\text {total }}\right)=N(\mathrm{HI})+2 N\left(\mathrm{H}_{2}\right) \approx 21 \times 10^{21} \mathrm{~cm}^{-2}$. This is a factor of $\sim 3$ larger than the Hi column that surrounds the molecular clumps but where no $\mathrm{CO}$ is detected. Therefore, the total $\left(\mathrm{HI}+2 \mathrm{H}_{2}\right)$ hydrogen column-density map exhibits a jump rather than a smooth transition whenever molecular gas is present. This sharp edge cannot be softened by a variation of the $X_{\mathrm{CO}}$ factor. Fukui et al. (1999) and Israel (1997), for example, find that the LMC has an $X_{\mathrm{CO}}$ factor a few times larger than the Galactic value. Using such a higher $X_{\mathrm{CO}}$ would result in larger $\mathrm{H}_{2}$ columns and therefore in an even steeper rise of total hydrogen column densities. If the $X_{\mathrm{CO}}$ factor is changed to produce smooth hydrogen maps, where the $\mathrm{H}_{2}$ traced by $\mathrm{CO}$ fills in the 'holes' of $\mathrm{HI}$, one requires an $X_{\mathrm{CO}}$ of about 3 times less than the Galactic value. No study of the LMC or of any other low metallicity object has shown such low $X_{\mathrm{CO}}$ factors before.

This comparison, however, is only valid if all molecular gas is traced by CO. If there are extended regions with molecular gas that is not traced by $\mathrm{CO}$, e.g. at low densities when $\mathrm{CO}$ may be dissociated by the surrounding radiation field but $\mathrm{H}_{2}$ is not, the sharp contrast in the 


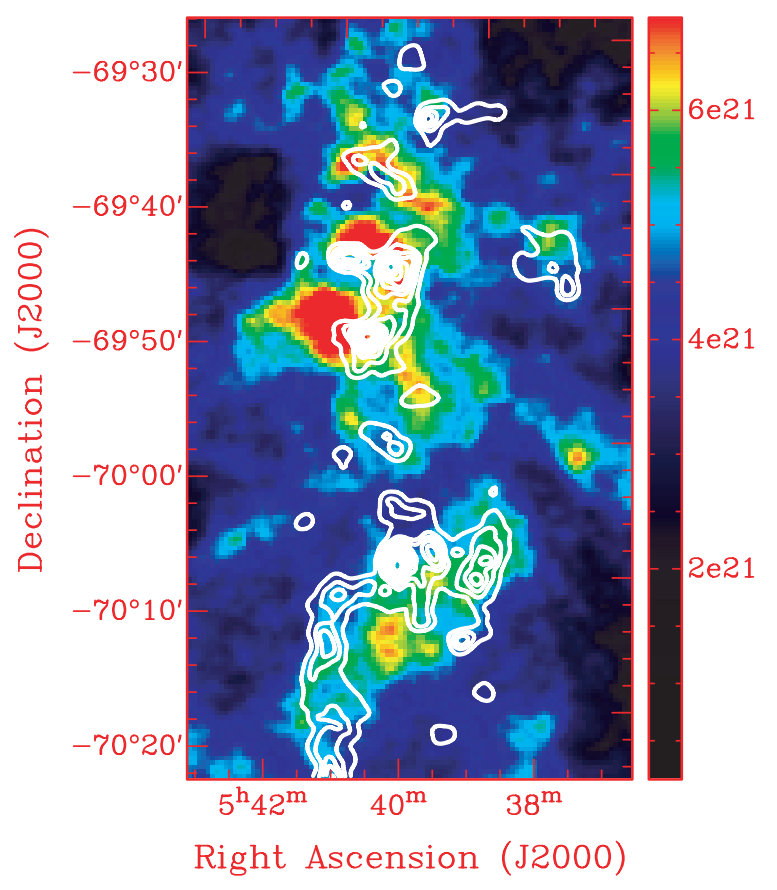

Figure $4 \mathrm{CO}$ contours of the region close to N159 overlaid as contours on an integrated Hi column-density map. Note that the CO peaks are close to but never coincident with nearby peaks of HI.

total hydrogen map may be smoothed out. Such an $\mathrm{H}_{2}$ phase is hard to detect and one of the best approaches is UV absorption spectroscopy. Tumlinson et al. (2002) performed such a survey toward many sightlines in the LMC with FUSE. They find that the molecular hydrogen, with a fraction of $\sim 1 \%$ (cf. Galaxy $\sim 10 \%$ ), is not a huge contributor to the entire gaseous ISM (see also the ORFEUS UV absorption observation presented in de Boer et al. 1998b). They were also observing sightlines close to 30 Dor where the UV radiation is very strong. If there are large amounts of $\mathrm{H}_{2}$ that are not traced by $\mathrm{CO}$ due to the higher dissociation energy of $\mathrm{H}_{2}$ they should be most obvious toward that region. Indeed, the total column of $\mathrm{H}_{2}$ they find close to 30 Dor is with $\sim 10^{20} \mathrm{~cm}^{-2}$ one of the largest in their survey. However, the Hi column density towards the same sightline is $\sim 70$ times larger. Similar fractions are found all across the LMC and the column of $\mathrm{H}_{2}$ in absorption never exceeds that of HI. It is therefore unlikely that the contrast between the $\mathrm{HI}$ and $\mathrm{H}_{2}$ column densities as traced by $\mathrm{CO}$ could be smoothed out with extended layers of molecular gas that are undetected in $\mathrm{CO}$ observations. This is in contrast to studies of dust extinction which suggest that there may be large, not CO-traced, reservoirs of molecular gas in the LMC (e.g. Imara \& Blitz 2007).

To conclude, the shifts of the HI and the $\mathrm{CO}$ peaks are probably not simply a result of warm hydrogen atoms (WNM) combining into $\mathrm{H}_{2}$. However, gravitation may accumulate a lot of material quickly in the densest molecular cores. Typical shifts between $\mathrm{CO}$ and $\mathrm{HI}$ peaks are of order $2^{\prime}$, which corresponds to $\sim 30 \mathrm{pc}$. Within a typical free fall time of a Myr, self-gravitation would accelerate
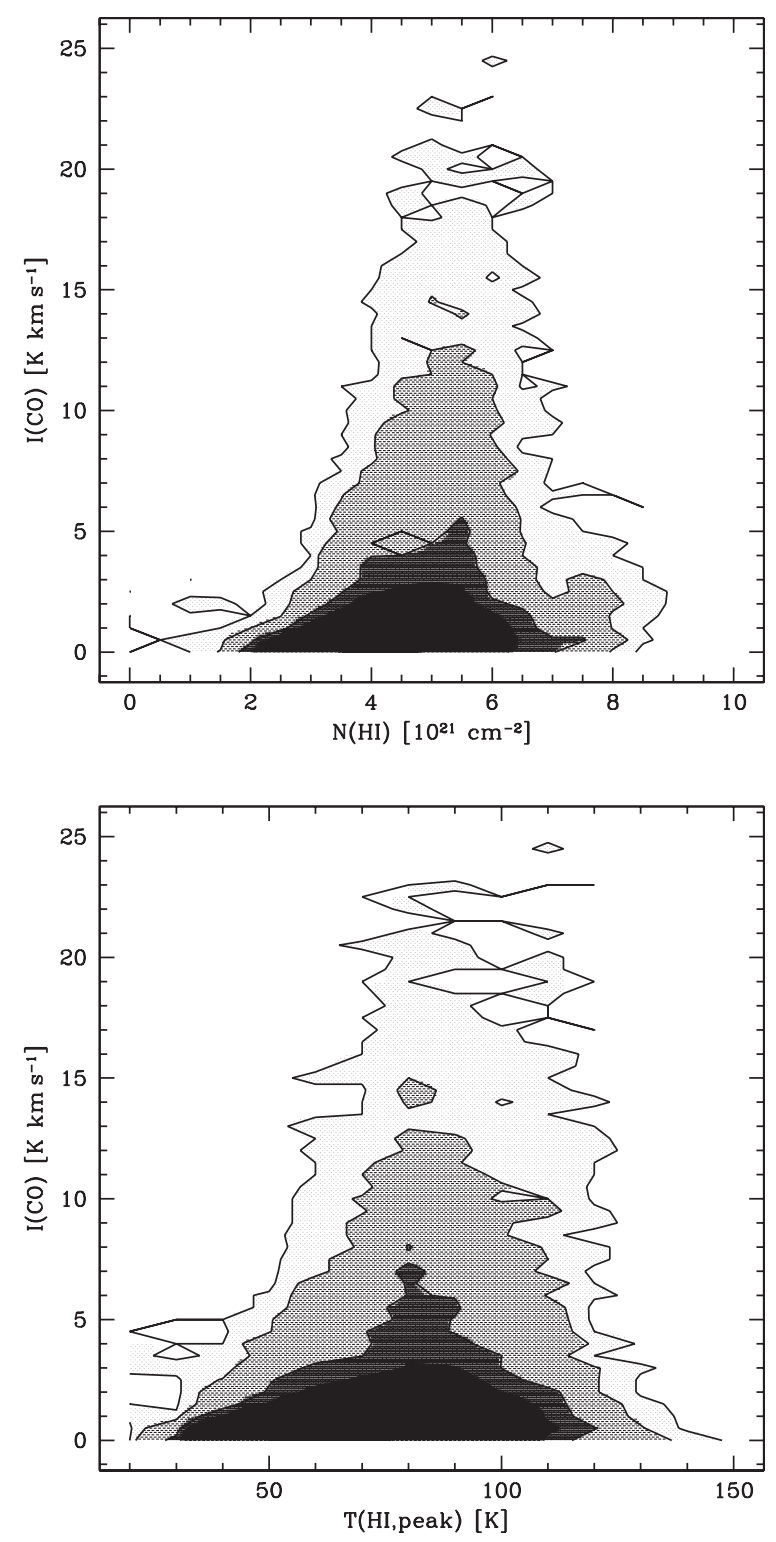

Figure 5 Pixel-based scatter plots of the ${ }^{12} \mathrm{CO}(1-0)$ luminosity as a function of Hi column density (top) and Hi peak brightness (bottom). The contours are levels of constant point density in logarithmic spacings.

the gas to a velocity of about $30 \mathrm{~km} \mathrm{~s}^{-1}$, a velocity gradient that is not ruled out by the position velocity diagrams displayed in Figure 3.

\subsubsection{Conversion of WNM to CNM to $\mathrm{H}_{2}$}

As shown in Figure 5, the Hi brightness temperature, at the location where most and the brightest molecular material resides, falls into the range of 60 to $100 \mathrm{~K}$ which corresponds to the kinetic temperature of the cold neutral medium in the Galactic disk (CNM, e.g., Dickey \& Lockman 1990). A conversion of WNM to CNM can be achieved by a shock. Indeed, the atomic gas to the east of the molecular ridge is prone to ram pressure effects as this side is the leading edge towards the Milky Way halo. The region is also influenced by tidal effects (e.g. StaveleySmith et al. 2003; Mastropietro et al. 2005) and it has 

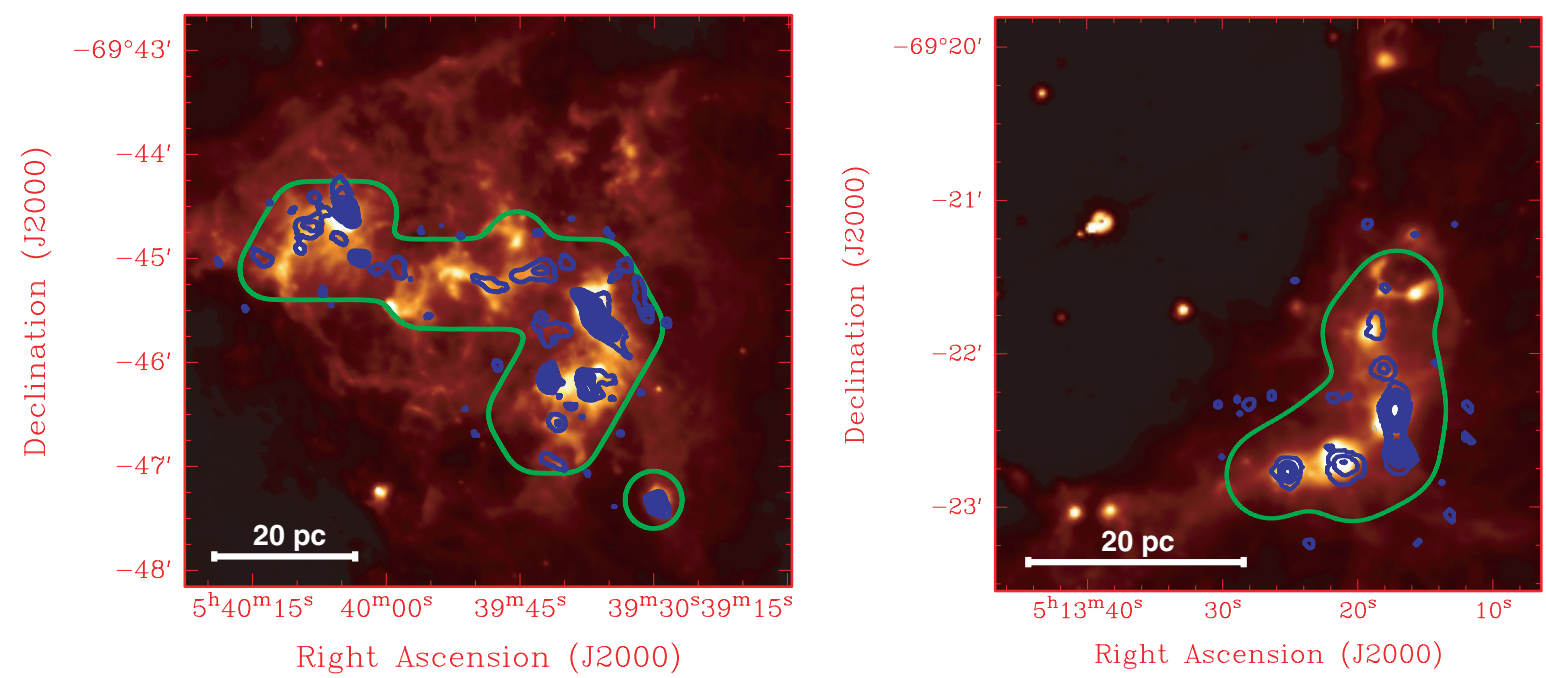

Figure 6 The star-forming regions N159 (left) and N113 (right) in the LMC. The contours show the integrated $\mathrm{HCO}^{+}(1-0)$ emission as observed with the ATCA on top of Spitzer 8- $\mu$ m maps. The green lines outline the mapped regions.

even been speculated that this region is responsible for the origin of the Magellanic Stream (Nidever, Majewski \& Burton 2008). The second moment map of the HI in Figure 1 is in fact not dominated by the intrinsic line width of the neutral gas but by the separation of disk and $L$ velocity components. At the eastern edge the separation is very large and the two components become closer in velocity space toward the western direction. At the position of the molecular ridge the disk and $L$ components start to merge. To date the origin of the bifurcation is still under discussion (e.g. Bekki \& Chiba 2007; Nidever, Majewski \& Burton 2008) but whether it is ram pressure or tidal effects or both, the shear and shocks from such an interaction introduces energy into the ISM that may reduce the likeliness to form molecular clouds. Indeed, some molecular complexes, the 'arc' clouds, are found in the region (Mizuno et al. 2001) but the bulk of molecular gas, the molecular ridge, is found where the disk and $L$ components start to merge into a single velocity component. If this indicates that they are also spatially merging, one may speculate that only the combination of both components can provide enough shielding for the gas to cool down and to become molecular.

In other words, shocks and/or shearing forces propagating from the eastern edge may convert some of the WNM into CNM. When the disk and $L$ component merge they may provide enough shielding to convert part of the CNM into molecular gas and the remaining, cold HI becomes optically thick and thus levels off at a brightness temperature close to the kinetic temperature of the CNM.

\subsection{3 $H_{I}$ Accretion}

The above process does not have to be static. As discussed earlier, we cannot rule out gas moving at speeds that are required to match free-fall timescales. Hi accretion after the formation of the first molecular cores may be responsible for the pile-up of neutral gas close to the molecular clouds. In this scenario, once the atomic gas comes close to the molecular clumps, the gas is shielded, cooled, and converted into $\mathrm{H}_{2}$ (see also Fukui 2007). HI accretion may therefore be described as a cooling flow. Cooling flows would result in decreasing $\mathrm{HI}$ line widths toward the molecular cores. We do not observe this on the scales of the HI-CO peak offsets but the most significant cooling may happen only very close to the molecular cores, too close to be resolved with the Australia Telescope Compact Array (ATCA) Hi observations at $21 \mathrm{~cm}$.

\subsubsection{Mechanical Feedback from Massive Stars}

Newly formed, massive stars deposit large amounts of energy into the surrounding interstellar medium (ISM) in the form of strong stellar winds and supernovae. Virtually all star-forming galaxies exhibit an ISM that is dominated by a wealth of expanding shells of all sizes. Kim et al. (2007) catalog the expanding shells in the LMC. Along the molecular ridge, the number density of the shells seems to be lower than in the rest of the LMC. It should therefore not have a significant effect on the distribution of the atomic and molecular gas. For some regions like the N159 region, however, some shell-like structure is observed in $\mathrm{HI}$ and dust maps (cf. Figure 6) and the $\mathrm{CO}$ emission toward this position may coincide with the inner side of the ring, similar to what is observed toward other expanding Galactic shells or LMC4 (Yamaguchi et al. 2001; Cappa et al. 2005).

The scenario that best fits the observations is the second one, in which shocks transform WNM into optically thick CNM followed by conversion into $\mathrm{H}_{2}$, with or without Hi accretion. The very sharp rise of the total hydrogen column density inferred at the position of the molecular gas is likely an artifact of high Hi optical depth. On the other hand, the confinement of CO emission to clumpy structures suggests an important role for self-gravity in shaping the molecular clouds. 


\subsection{Star Formation}

Typical Hi column-density thresholds in massive and dwarf galaxies, above which SF is observed, hover around a canonical value of $\sim 1 \times 10^{21} \mathrm{~cm}^{-2}$ (e.g. Walter et al. 2007). Below this threshold SF appears to be suppressed. For most of the LMC this rule holds well. However, the south-eastern region, dominated by ram pressure and tidal forces of the LMC/SMC/Milky Way interaction, appears to be different. Virtually no SF, as traced by $\mathrm{H} \alpha$, is observed in this region at positions where the Hi column density is below $5 \times 10^{21} \mathrm{~cm}^{-2}$. This may be due to the tidal and ram pressure forces in the two gas components of the south-eastern LMC.

On smaller scales, SF occurs in the very densest molecular clumps. We conducted a survey with the ATCA of the high-density gas tracers $\mathrm{HCO}^{+}(1-0)$ and $\mathrm{HCN}(1-0)$ towards the star-forming regions N159 (40 pointing mosaic) in the molecular ridge and toward N113 (7 pointing mosaic) at the western tip of the LMC bar. The observations have a resolution of $\sim 7^{\prime \prime}$ which, at the distance of the LMC, corresponds to a physical length of $\sim 1.7$ pc. Both of the observed molecules are only excited at volume densities exceeding $\sim 10^{4} \mathrm{~cm}^{-3}$. $\mathrm{HCO}^{+}$, however, is also a tracer for photo-dominated regions and can also form in more diffuse material where the $\mathrm{C}^{+}$abundance is high. Integrated $\mathrm{HCO}^{+}$intensity maps overlaid on $8-\mu \mathrm{m}$ Spitzer maps are shown in Figure 6. In both regions, the dense molecular gas typically overlaps or is adjacent to bright infrared (IR) emission peaks. These peaks are the locations where individual stars and stellar clusters eventually form within the larger molecular complexes traced by $\mathrm{CO}$. Comparing the $\mathrm{HCN}$ and $\mathrm{HCO}^{+}$ maps, we find that, after beam deconvolution, the $\mathrm{HCO}^{+}$ emission is about $20-30 \%$ more extended than the $\mathrm{HCN}$ clumps, in agreement with the results presented in Wong et al. (2006). The 8- $\mu \mathrm{m}$ IR morphology of N159 appears to be ring-like and indeed it is a 1 to $2-\mathrm{Myr}$-old windblown bubble created by a central, massive star (Jones et al. 2005). Most of the dense molecular gas is situated at the rim of this shell, with relatively little observed at projected sightlines towards the interior. N113, in contrast, is a long, bent string of several individual knots, both visible in the IR and in the $\mathrm{HCO}^{+}$and $\mathrm{HCN}$ maps. The string of knots curves from the south-east towards the north. This morphology appears reminiscent of a circular arc, and close to the center of this hypothetical circle, towards the north-east, one indeed finds HD269219, a 30-M $\odot$ supergiant B star and HD269217, an emission-line star. Thus, the densest pockets of molecular gas and the associated star-forming regions appear to be located on a shock front created by the two energy-injecting massive stars, not unlike the situation of the shell in N159.

The two examples, and the above discussion on molecular cloud formation argues that shocks are the major mechanism to trigger both, the formation of low-density molecular clouds and the formation of star-forming, highdensity molecular clumps within these larger structures.

\section{Summary}

The LMC is a unique object with which to study molecular cloud and star formation on galaxy-wide scales with high spatial detail and in a large range of very different environments. A very prominent molecular feature stretches from 30 Dor $1.8 \mathrm{kpc}$ southward; it contains about one third of the entire molecular content of the LMC, as traced by CO. Our Mopra data of this molecular ridge reveals the following:

- The structure is most likely not a coherent, large molecular complex but consists of smaller molecular clouds that are observed to be embedded in different velocity components of the neutral atomic gas. However, the global picture of the LMC shows that the molecular ridge is at the innermost edge of the south-eastern highdensity region of the LMC, a region where tidal and ram pressure forces have a major influence on the state of the gas. The ridge coincides with $\mathrm{HI}$ at high column density and is just where the disk and $L$ Hi velocity components start to merge. Thus, the molecular clouds in the ridge are likely to share the same formation histories and timescales.

- The local Hi and CO peaks are typically displaced by $\sim 30$ pc. They are usually near each other but hardly ever coincident. A likely explanation for this morphology is that shocks propagate from the eastern edge of the LMC and turn warm Hi into partly optically thick, cold Hi and eventually into molecular hydrogen. This may happen in a static sense or via Hi accretion. The hydrogen column densities within the molecular clumps are much larger than in the surrounding material, which indicates that the clouds are self-gravitating. Other mechanisms, such as feedback from massive stars may also be responsible for the shift of $\mathrm{HI}$ and $\mathrm{CO}$ peaks but are likely only playing a role for a small number of molecular complexes.

- Very dense pockets of molecular gas, as traced by $\mathrm{HCO}^{+}(1-0)$ and $\mathrm{HCN}(1-0)$, are found near and coinciding with strong IR emission. This strengthens the suggested correlation of the very dense gas and actual star formation rates. For the N159 and N113 regions we find the high density gas peaks to be mostly located on shock fronts and on the rims of expanding shells, powered by central, massive stars.

Over the coming years, the study of molecular gas in the LMC and SMC enters a new era. Our team will continue to map all the molecular gas in both systems with the Mopra (MAGMA survey) and ATCA telescopes. In addition, many new facilities are currently under construction or have just been completed that can be used to observe the LMC, such as APEX, ASTE, NANTEN2, ALMA, as well as satellites such as Herschel and Spitzer. Studying molecular gas in the LMC provides a unique link between Galactic and extragalactic observations and will be germane to the full understanding of star formation on all scales. 


\section{Acknowledgments}

Jürgen Ott is a Jansky Fellow of the National Radio Astronomy Observatory. Erik Muller is a Bolton Fellow. The Australia Telescope Compact Array is part of the Australia Telescope which is funded by the Commonwealth of Australia for operation as a National Facility managed by CSIRO.

\section{References}

Bekki, K. \& Chiba, M., 2007, PASA, 24, 21

Braun, J. M., Bomans, D. J., Will, J.-M. \& de Boer, K. S., 1997, A\&A, 328, 167

Cappa, C., Niemela, V. S., Martín, M. C. \& McClure-Griffiths, N. M., 2005, A\&A, 436, 155

Cohen, R. S., Dame, T. M., Garay, G., Montani, J., Rubio, M. \& Thaddeus, P., 1988, ApJ, 331, L95

Cohen, M., Staveley-Smith, L. \& Green, A., 2003, MNRAS, 340,275

Connors, T. W., Kawata, D. \& Gibson, B. K., 2006, MNRAS, 371,108

de Boer, K. S., Richter, P., Bomans, D. J., Heithausen, A. \& Koorneef, J., 1998a, A\&A, 338, L5

de Boer, K. S., Braun, J. M., Vallenari, A. \& Mebold, U., 1998b, A\&A, 329, L49

Dickey, J. M. \& Lockman, F. J., 1990, ARA\&A, 28, 215

Fujimoto, M. \& Noguchi, M., 1990, PASJ, 42, 505

Fukui, Y. et al., 1999, PASJ, 51, 745

Fukui, Y., 2007, in IAU Symposium 237, Triggered Star Formation in a Turbulent ISM, Eds. Elmergreen, B. G. \& Palous, J., 31
Gardiner, L. T., Sawa, T. \& Fujimoto, M., 1994, MNRAS, 266, 567

Gardiner, L. T., Turfus, C. \& Putman, M. E., 1998, ApJ, 507, L35

Imara, N. \& Blitz, L., 2007, ApJ, 662, 969

Israel, F. P., 1997, A\&A, 328, 471

Johansson, L. E. B. et al., 1998, A\&A, 331, 857

Jones, T. J., Woodward, C. E., Boyer, M. L., Gehrz, R. D. \& Polomski, E., 2005, ApJ, 620, 731

Kim, S., Staveley-Smith, L., Dopita, M. A., Freeman, K. C., Sault, R. J., Kesteven, M. J. \& McConnell, D., 1998, ApJ, 503, 674

Kim, S. et al., 2007, ApJS, 171, 419

Kutner, M. L. et al., 1997, A\&AS, 122, 255

Ladd, N., Purcell, C., Wong, T. \& Robertson, S., 2005, PASA, 22, 62

Luks, T. \& Rohlfs, K., 1992, A\&A, 263, 41

Mastropietro, C., Moore, B., Mayer, L., Wadsley, J. \& Stadel, J., 2005, MNRAS, 363, 509

Mizuno, N. et al., 2001, PASJ, 53, 971

Murai, T. \& Fujimoto, M., 1980, PASJ, 32, 581

Nidever, D. L., Majewski, S. R. \& Burton, W. B., 2008, ApJ, 679, 432

Pineda, J. L., Ott, J., Klein, U., Wong, T., Muller, E. \& Hughes, A., 2008, ApJ, submitted

Staveley-Smith, L., Kim, S., Calabretta, M. R., Haynes, R. F. \& Kesteven, M. J., 2003, MNRAS, 339, 87

Strong, A. W. et al., 1988, A\&A, 207, 1

Tumlinson, J. et al., 2002, ApJ, 566, 857

van der Marel, R. P. \& Cioni, M.-R. L., 2001, AJ, 122, 1807

Walter, F. et al., 2007, ApJ, 661, 102

Wong, T., Whiteoak, J. B., Ott, J., Chin, Y. \& Cunningham, M. R., 2006, ApJ, 649, 224

Yamaguchi, R., Mizuno, N., Onishi, T., Mizuno, A. \& Fukui, Y., 2001, ApJL, 553, L185 